\title{
Increased level of apolipoprotein B/apolipoprotein A1 ratio as a potential risk for osteonecrosis
}

Keita Miyanishi, Takuaki Yamamoto, Takahiko Irisa, Yasuo Noguchi, Yoichi Sugioka, Yukihide Iwamoto index, ${ }^{4}$ clinical history, electrocardiogram, and haematological and urinary examinations.

Objective-This study was performed to investigate whether a high ratio of apolipoprotein B to apolipoprotein A1 (apo $B / a p o$ A1 ratio) is significantly associated with the risk of developing non-traumatic osteonecrosis of the femoral head (ON). Methods-Fifty consecutive nontraumatic ON cases were compared with 50 age and sex matched controls, using both univariate and stepwise discriminant analyses, regarding the factors of corticosteroid, alcohol, cigarettes, cholesterol, triglyceride, and apo $B /$ apo $A 1$ ratio. To eliminate the possibility that $\mathrm{ON}$ or osteoarthritic change itself can increase the apo B/apo $A 1$ ratio, a further 32 consecutive cases comprising nine traumatic $O N$ and 23 osteoarthritis (OA) patients were analysed using Scheffe's test.

Results-There was a significant association between a high apo B/apo $A 1$ ratio and the development of non-traumatic ON with both univariate $(p=0.0001)$ and stepwise discriminant analyses (partial $\left.r^{2}=0.1239, p=0.0004\right)$. The apo B/apo A1 ratio in the non-traumatic ON group was significantly higher than that in the traumatic ON $(p<0.01)$, control $(p<0.001)$, or the OA groups $(\mathrm{p}<0.001)$.

Conclusion-A high apo $\mathrm{B} / \mathrm{apo} \mathrm{A} 1$ ratio is significantly associated with the risk of developing $O N$. This ratio may be useful for assessing the potential risk of developing osteonecrosis.

(Ann Rheum Dis 1999;58:514-516)

As a useful serological marker of cholesterol transport, the ratio of apolipoprotein $\mathrm{B}$ to apolipoprotein $\mathrm{A} 1$ (apo $\mathrm{B} / \mathrm{apo} \mathrm{A} 1$ ratio) has been emphasised mainly in the field of ischaemic heart disease. A high apo $\mathrm{B} / \mathrm{apo} \mathrm{A} 1$ ratio is considered to reflect prominent cholesterol transport to peripheral tissue. ${ }^{1}$

Osteonecrosis of the femoral head $(\mathrm{ON})$ is thought to be the result of ischaemic insult to bone and bone marrow tissue. ${ }^{23}$ This study was designed to determine whether a high apo $\mathrm{B} /$ apo $\mathrm{A} 1$ ratio is significantly associated with the risk of developing non-traumatic $\mathrm{ON}$.

Orthopaedic Surgery,

Faculty of Medicine,

Kyushu University,

3-1-1 Maidashi,

Higashi-ku, Fukuoka

812-8582, Japan

Correspondence to:

Dr T Yamamoto.

Accepted for publication 6 April 1999

\section{Non-traumatic ON group}

The non-traumatic ON group consisted of 50 consecutive Japanese patients, who had been referred to Kyushu University Hospital between August 1996 and September 1997 with the diagnosis of non-traumatic $\mathrm{ON}$, as confirmed by roentgenography, bone scintigram, magnetic resonance imaging (MRI), and histopathological examination. ${ }^{2} 35$

This group consisted of three subgroups, corticosteroid, alcohol, and idiopathic groups. The corticosteroid group (20 patients) had a history of corticosteroid treatment for underlying diseases. The alcohol group (18 patients) was defined based on a report that the consumption of over $400 \mathrm{ml}$ of ethanol per week results in a significant risk of developing ON. ${ }^{6}$ The remaining 12 patients were categorised as idiopathic, as they had no associated risk factors for $\mathrm{ON}^{2}$

\section{Control group}

This group consisted of 50 randomly selected healthy Japanese volunteers who had no history of corticosteroid use, alcohol abuse, or associated risk factors for $\mathrm{ON}$.

Traumatic ON group and osteoarthritis of the hip group

The traumatic ON group consisted of nine consecutive Japanese patients, who had been referred to our hospital during the same period. The osteoarthritis (OA) group consisted of 23 consecutive Japanese patients who had also been referred to our hospital during the same period, among whom secondary OA after collapsed $\mathrm{ON}$ had been excluded. ${ }^{7}$ Patients in both groups had no risk factors for ON.

\section{LABORATORY TESTS}

All preoperative blood samples were obtained from each of the $\mathrm{ON}$ and $\mathrm{OA}$ patients between 7 and $8 \mathrm{am}$, after a 12 hour recumbent fasting period. Blood samples from the controls were obtained under the same conditions. Total serum cholesterol and triglyceride were measured by enzymatic methods ${ }^{8}$ with high precision (coefficients of variation were $0.3 \%$ for cholesterol and $0.5 \%$ for triglyceride). Serum levels of apolipoprotein A1 (apo A1) and apolipoprotein B (apo B) were measured by turbidimetric immunoassay, ${ }^{9}$ with a coefficient of variation of $2.3 \%$ for apo A1 and $1.1 \%$ for apo B.
PATIENTS AND CONTROLS

this study, none of the patients had any tory of obesity, ischaemic heart disease, hypertension, diabetes mellitus, or the use of diuretics or $\beta$ blockers, as verified by body mass 
Table 1 Univariate (top) and multivariate (bottom) analyses of risk factors for developing osteonecrosis of the femoral head

\begin{tabular}{|c|c|c|c|}
\hline \multirow[b]{2}{*}{ Risk factor * } & \multicolumn{2}{|l|}{$O N$} & \multirow[b]{2}{*}{$p t$} \\
\hline & $+(n=50)$ & $-(n=50)$ & \\
\hline \multicolumn{4}{|l|}{ Corticosteroid } \\
\hline+ & 20 & 0 & \\
\hline- & 30 & 50 & 0.0001 \\
\hline \multicolumn{4}{|l|}{ Sex } \\
\hline male & 34 & 34 & \\
\hline female & 16 & 16 & 1.0000 \\
\hline Age $(y)$ & $44.4 \quad(14.9)$ & $44.4 \quad(14.5)$ & 1.0000 \\
\hline Alcohol (ml/week $) \ddagger$ & $388.0 \quad(401.0)$ & $82.9 \quad(142.0)$ & 0.0001 \\
\hline Cigarettes (no/day) & $17.6(19.0)$ & 7.4 (13.3) & 0.0027 \\
\hline Cholesterol (mg/dl) & 192.7 & 203.8 & 0.1081 \\
\hline Triglyceride $(\mathrm{mg} / \mathrm{dl})$ & $121.9 \quad(39.9)$ & 109.9 (46.1) & 0.1685 \\
\hline Apo $\mathrm{B} /$ apo $\mathrm{A} 1$ ratio & $0.85(0.24)$ & $0.62(0.18)$ & 0.0001 \\
\hline Risk factor & partial $r^{2} \int$ & & $p S$ \\
\hline Corticosteroid & 0.2500 & & 0.0001 \\
\hline Alcohol & 0.3163 & & 0.0001 \\
\hline Apo B/apo A1 ratio & 0.1239 & & 0.0004 \\
\hline Cigarettes & 0.0005 & & 0.8327 \\
\hline Cholesterol & 0.0067 & & 0.4268 \\
\hline Triglyceride & 0.0000 & & 0.9938 \\
\hline
\end{tabular}

${ }^{\star}$ Corticosteroid and sex are listed as numbers of subjects. The other factors are given as means (SD). +Univariate analyses were performed by means of Fisher's exact test for corticosteroid, $\chi$ test for sex, and Student's $t$ tests for age, alcohol, cigarettes, cholesterol, triglyceride, and the apo $\mathrm{B} /$ apo $\mathrm{A} 1$ ratio. $¥$ Values are given as weekly ethanol consumption. \Stepwise discriminant analysis was used. Apo B/apo A1 ratio = apolipoprotein B/apolipoprotein A1 ratio; $\mathrm{ON}=$ osteonecrosis of the femoral head. patients had received corticosteroid treatment for systemic lupus erythematosus, three for aplastic anaemia, three for nephrosis, two for idiopathic thrombocytopenic purpura, one for uveitis, one for rheumatoid arthritis, and one for adrenal dysfunction. The alcohol group consisted of 18 men, ranging from 26 to 67 years of age (mean (SD) 44.0 (9.8) years), the idiopathic group consisted of seven men and five women, ranging from 26 to 73 years of age (mean (SD) 51.0 (18.5) years), and the traumatic ON group consisted of five men and four women, ranging from 12 to 45 years of age (mean (SD) 30.3 (10.9) years), of whom seven patients had antecedent femoral neck fracture, one had femoral head fracture, and one had fracture dislocation of the hip.

The control group consisted of 34 men and 16 women, ranging from 20 to 78 years of age (mean (SD) 44.4 (14.5) years), while the OA group consisted of seven men and 16 women, ranging from 22 to 74 years of age (mean (SD) 45.7 (16.5) years).

ASSOCIATION BETWEEN RISK FACTORS AND NON-TRAUMATIC ON

Table 1 summarises the results of univariate and multivariate analyses. Those factors that were significantly associated with nontraumatic $\mathrm{ON}$ in the multivariate analysis were corticosteroid, alcohol, and the apo B/apo A1 ratio.

differences in proportions of corticosteroid history and sex, respectively, and Student's $t$ tests for numerical data of age, alcohol, cigarettes, cholesterol, triglyceride, and the apo B/apo A1 ratio. Factors except for those with a $\mathrm{p}$ value of 1.0000 were further analysed by multivariate analysis using stepwise discriminant analysis, ${ }^{10}$ where adjustments for all confounding factors including corticosteroid, alcohol, and age were performed.

To eliminate the possibility that $\mathrm{ON}$ or $\mathrm{OA}$ itself can increase the level of the apo B/apo A1 ratio, multiple comparisons of the apo B/apo A1 ratio were performed using Scheffe's test with $\mathrm{p}<0.05$ being considered significant.

All the analyses were performed with the SAS software (SAS Institute Ltd, Tokyo, Japan).

\section{Results}

CHARACTERISTICS OF THE STUDY SAMPLE

The mean (SD) age in the non-traumatic ON group was 44.4 (14.9) years. The corticosteroid group consisted of nine men and 11 women, ranging from 20 to 70 years of age (mean (SD) 40.9 (15.9) years), of whom nine

Table 2 Multiple comparisons of apolipoprotein B lapolipoprotein $A 1$ ratio

\begin{tabular}{ll}
\hline Group & $\begin{array}{l}\text { Apolipoprotein B } \\
\text { lapolipoprotein A1 ratio }\end{array}$ \\
\hline Non-traumatic ON & $0.85(0.24)$ \\
Corticosteroid & $0.85(0.20)$ \\
Alcohol & $0.86(0.29)$ \\
Idiopathic & $0.84(0.24)$ \\
Control & $0.62(0.18)^{\star}$ \\
Traumatic ON & $0.59(0.16)^{\star \star}$ \\
OA & $0.63(0.18)^{\star}$ \\
\hline
\end{tabular}

Values are means $(\mathrm{SD}) . \mathrm{ON}=$ osteonecrosis of the femoral head; OA $=$ osteoarthritis. ${ }^{\star} \mathrm{p}<0.001,{ }^{\star \star} \mathrm{p}<<0.01$ compared with non-traumatic ON
MULTIPLE COMPARISONS OF THE APO B/APO A1 RATIO BETWEEN THE SUBGROUPS

The apo B/apo $\mathrm{A} 1$ ratio in the non-traumatic ON group was significantly higher than that in the traumatic ON $(p<0.01)$, control $(p<0.001)$, or the OA groups $(\mathrm{p}<0.001)$, but there was no significant difference between the latter three groups. The apo B/apo A1 ratio was not significantly different between the corticosteroid, alcohol, and idiopathic groups (table 2).

\section{Discussion}

Patients with a history of corticosteroid treatment or alcohol abuse have a higher risk of developing ON compared with healthy people, ${ }^{2}$ and as such it is not uncommon for cases initially diagnosed as not having ON based on MRI or biopsy to then develop ON several months later. It is thus difficult to define the end point at which there is no risk of suffering from $\mathrm{ON}$ in such cases. To exclude such false negative cases, patients who had a history of corticosteroid treatment or alcohol abuse were not included in the control group in this study.

Multivariate analysis proved that the apo $\mathrm{B} /$ apo $\mathrm{A} 1$ ratio was associated with the risk of ON independently of corticosteroid and alcohol. This view is supported by several clinical reports. The apo B/apo A1 ratio in SLE patients has been reported to show no remarkable change after corticosteroid treatment. ${ }^{11} \mathrm{~A}$ low apo B/apo $\mathrm{A} 1$ ratio has been reported in alcoholics. ${ }^{12}$ We thus conclude that the apo B/apo A1 ratio had actually been raised from the outset in the corticosteroid and alcohol ON cases. 
The apo $\mathrm{B} / \mathrm{apo} \mathrm{A} 1$ ratio has been reported to increase gradually over a lifetime. In the multiple comparison, the ages in each group were not exactly matched. The mean age in the idiopathic ON group was seven years older than that in the control group, however, a span of seven years around the age of 50 seems to show no significant difference in the level of the apo $\mathrm{B} /$ apo A1 ratio. ${ }^{13}$ The mean age in the traumatic $\mathrm{ON}$ group was 14 years younger than that in the non-traumatic ON group. The traumatic ON group was included to refute the hypothesis that an increased apo B/apo A1 ratio is the result of $\mathrm{ON}$. We therefore believe that the non-increased apo B/apo $\mathrm{A} 1$ ratio in the traumatic ON group was meaningful in that it could at least refute such a hypothesis despite the difference in age.

The abnormal lipid deposition in bone and bone marrow tissue that has been reported both in human $\mathrm{ON}$ cases $^{14}$ and in an animal model,${ }^{15}$ may conceivably be related to prominent lipid transport to bone tissue resulting from a high apo $\mathrm{B} / \mathrm{apo} \mathrm{A} 1$ ratio. It is our belief that the apo B/apo A1 ratio may be useful not only for assessing the potential risk of developing $\mathrm{ON}$, but also for exploring the possible mechanism of $\mathrm{ON}$.

We thank Mr Noriya Taki (Statistical Analysis of Medicine, Fukuoka, Japan) for his useful advice on the statistical analysis. The English used in this manuscript was revised by Miss K Miller (Royal English Language Centre, Fukuoka, Japan). Funding: this study was supported by a Grant for Research on Funding: this study was supported by a Grant for Research on plantation from the Ministry of Health and Welfare of Japan and plantation from the Ministry of Health and
by the Hip Joint Foundation of Japan, Inc.
1 Srinivasan SR, Berenson GS. Serum apolipoproteins A-I and $\mathrm{B}$ as markers of coronary artery disease risk in early life: The Bogalusa Heart Study. Clin Chem 1995;41:159-64.

2 Mankin HJ. Nontraumatic necrosis of bone (Osteonecrosis). N Engl J Med 1992;326:1473-9.

3 Yamamoto T, Irisa T, Sugioka Y, Sueishi K. Effects of pulse methylprednisolone on bone and marrow tissues. Arthritis Rheum 1997;40:2055-64.

4 Bray GA. Definition, measurement, and classification of the syndromes of obesity. Int J Obes 1978;2:99-112.

5 Kubo T, Yamazoe S, Sugano N, Fujioka M, Naruse S, Yoshimura $\mathrm{N}$, et al. Initial MRI findings of non-traumatic osteonecrosis of the femoral head in renal allograft recipients. Magn Reson Imaging 1997;15:1017-23.

6 Matsuo K, Hirohata T, Sugioka Y, Ikeda M, Fukuda A. Influence of alcohol intake, cigarette smoking, and occupational status on idiopathic osteonecrosis of the femora head. Clin Orthop 1988;234:115-23.

7 Macys JR, Bullough PG, Wilson PD Jr. Coxarthrosis: A study of the natural history based on a correlation of clinical, radiographic, and pathologic findings. Semin Arthritis Rheum 1980;10:66-80.

8 Allain CC, Poon LS, Chan CSG, Richmond W, Fu PC. Enzymatic determination of total serum cholesterol. Clin Chem 1974;20:470-5.

9 Noma A, Hata Y, Goto Y. Quantitation of serum apolipoprotein A-I, A-II, B, C-II, C-III and E in healthy Japanese by turbidimetric immunoassay: reference values, and age- and sex-related differences. Clin Chim Acta 1991; 199:147-58.

10 Lachenbruch PA. Discriminant analysis. New York: Hafner, 1975:29-36.

11 Ilowite NT, Samuel P, Ginzler E, Jacobson MS. Dyslipoproteinemia in pediatric systemic lupus erythematosus. Arthritis Rheum 1988;31:859-63.

12 Barboriak JJ, Alaupovic P, Cushman P. Abstinence-induced changes in plasma apolipoprotein levels of alcoholics. Drug Alcohol Depend 1981;8:337-43.

13 Avogaro P, Cazzolato G, Bittolo BG, Belussi F, Quinci GB Values of apo-AI and apo-B in humans according to age and sex. Clin Chim Acta 1979;95:311-15.

14 Boskey AL, Raggio CL, Bullough PG, Kinnett JG. Changes in the bone tissue lipids in persons with steroid- and alcohol-induced osteonecrosis. Clin Orthop 1983;172: 289-95.

15 Kawai K, Maruno H, Watanabe Y, Hirohata K. Fat necrosis of osteocytes as a causative factor in idiopathic osteonecrosis in heritable hyperlipemic rabbits. Clin Orthop 1980; 153:273-82. 